\title{
Belgeo
}

Revue belge de géographie

\section{Vulnérabilité et résilience des socio-écosystèmes littoraux d'Afrique de l'Ouest : état des connaissances actuelles et interrogation sur le devenir du littoral sénégalo-bissau-guinéen}

Vulnerability and resilience of West African coastal socio-ecosystems: current knowledge and questions about the future of the Bissau-Guinea-Senegal coastal area

Tidiane Sané, El Hadji Balla Dièye, Boubacar Solly, Boubacar Demba Ba, Mamadou Thior, Luc Descroix, Marie-Christine Cormier-Salem et Mouhamadou Mawlid Diakhaté

\section{(2) OpenEdition}

\section{Journals}

Édition électronique

URL : https://journals.openedition.org/belgeo/50403

DOI : 10.4000/belgeo.50403

ISSN : 2294-9135

Éditeur :

National Committee of Geography of Belgium, Société Royale Belge de Géographie

Référence électronique

Tidiane Sané, El Hadji Balla Dièye, Boubacar Solly, Boubacar Demba Ba, Mamadou Thior, Luc Descroix Marie-Christine Cormier-Salem et Mouhamadou Mawlid Diakhaté, "Vulnérabilité et résilience des socio-écosystèmes littoraux d'Afrique de l'Ouest : état des connaissances actuelles et interrogation sur le devenir du littoral sénégalo-bissau-guinéen », Belgeo [En ligne], 1 | 2021, mis en ligne le 09 septembre 2021, consulté le 24 octobre 2021. URL : http://journals.openedition.org/belgeo/50403 DOl : https://doi.org/10.4000/belgeo.50403

Ce document a été généré automatiquement le 24 octobre 2021.

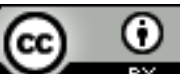

Belgeo est mis à disposition selon les termes de la licence Creative Commons Attribution 4.0 International. 


\title{
Vulnérabilité et résilience des socio- écosystèmes littoraux d'Afrique de l'Ouest : état des connaissances actuelles et interrogation sur le devenir du littoral sénégalo-bissau- guinéen
}

\author{
Vulnerability and resilience of West African coastal socio-ecosystems: current
} knowledge and questions about the future of the Bissau-Guinea-Senegal coastal area

Tidiane Sané, El Hadji Balla Dièye, Boubacar Solly, Boubacar Demba Ba, Mamadou Thior, Luc Descroix, Marie-Christine Cormier-Salem et Mouhamadou Mawlid Diakhaté

\section{Introduction}

L'Afrique de l'Ouest constitue une des plus importantes réserves de la biodiversité à l'échelle mondiale (Cormier-Salem, 1994). Elle abrite plusieurs régions littorales (de la Mauritanie au Nigéria) parcourues par un réseau de fleuves débouchant sur des estuaires particulièrement riches et diversifiés, surtout à l'ouest du fait des remontées d'eaux froides (upwelling) (Cormier-Salem, 1994 ; Faye, 2010). Le profil écologique des littoraux ouest-africains, particulièrement les deltas transfrontaliers (fleuve Sénégal, Saloum-Niumi, Casamance-Geba), est assez largement dominé par les écosystèmes de mangrove, indispensables pour la vie des communautés humaines qui vivent d'activités diverses: pêche, riziculture, tourisme, cueillette, chasse, apiculture, élevage, arboriculture, etc. Paradoxalement, ces littoraux comptent parmi les régions du monde les plus touchées par l'insécurité alimentaire, la pauvreté et la migration (GRDR et al., 
2017). De fortes menaces pèsent sur ces milieux littoraux : changement climatique, salinisation et acidification des terres, développement de pratiques minières, surexploitation des ressources naturelles, etc. Dans ce contexte, les conflits entre usagers des ressources naturelles (population locale, pêcheurs, gestionnaires des aires protégées, exploitants miniers, etc.) se développent davantage et conduisent parfois à des situations géopolitiques complexes.

2 La double exigence de lutte contre la dégradation de la biodiversité et de création de richesses pour les populations dépendantes des ressources des écosystèmes côtiers est un enjeu crucial pour l'Afrique de l'Ouest où les changements rapides de tous ordres sont notés (climatiques, démographiques, économiques, institutionnels). C'est dans ce contexte que le Laboratoire Mixte International « Patrimoines et Territoires de l'Eau (LMI-PATEO) », dans une perspective de prendre la mesure de ces enjeux et mutations, analyse ces environnements côtiers (fig. 1) soumis à de fortes mutations liées aux changements globaux. En inscrivant ces actions sur les dynamiques environnementales, économiques et sociétales sur les littoraux ouest-africains, des chercheurs du laboratoire ont tenté de documenter les enjeux complexes que représente le maintien des services écosystémiques en lien avec le bien-être des populations, et de s'interroger sur les valeurs de la biodiversité et le partage des avantages issus des socio-écosystèmes (Badiane, 2016; Descroix et al., 2016 ; CormierSalem et al., 2017a ; GRDR et al., 2017 ; Sané, 2017; Sané et al., 2018; Soumaré, 2018 ; Mendy, 2018; Thior et al., 2019; Thior, 2020; Diéye et al., 2021; Andrieu, 2021). À travers les travaux réalisés par ses chercheurs, PATEO a ainsi répondu aux priorités tant géopolitiques que scientifiques des États et des institutions de recherche, et a su contribuer à la co-construction des savoirs et à la coproduction des connaissances sur ces milieux riches, mais fragiles et vulnérables. 
Figure 1. Zones d'intervention du PATEO.

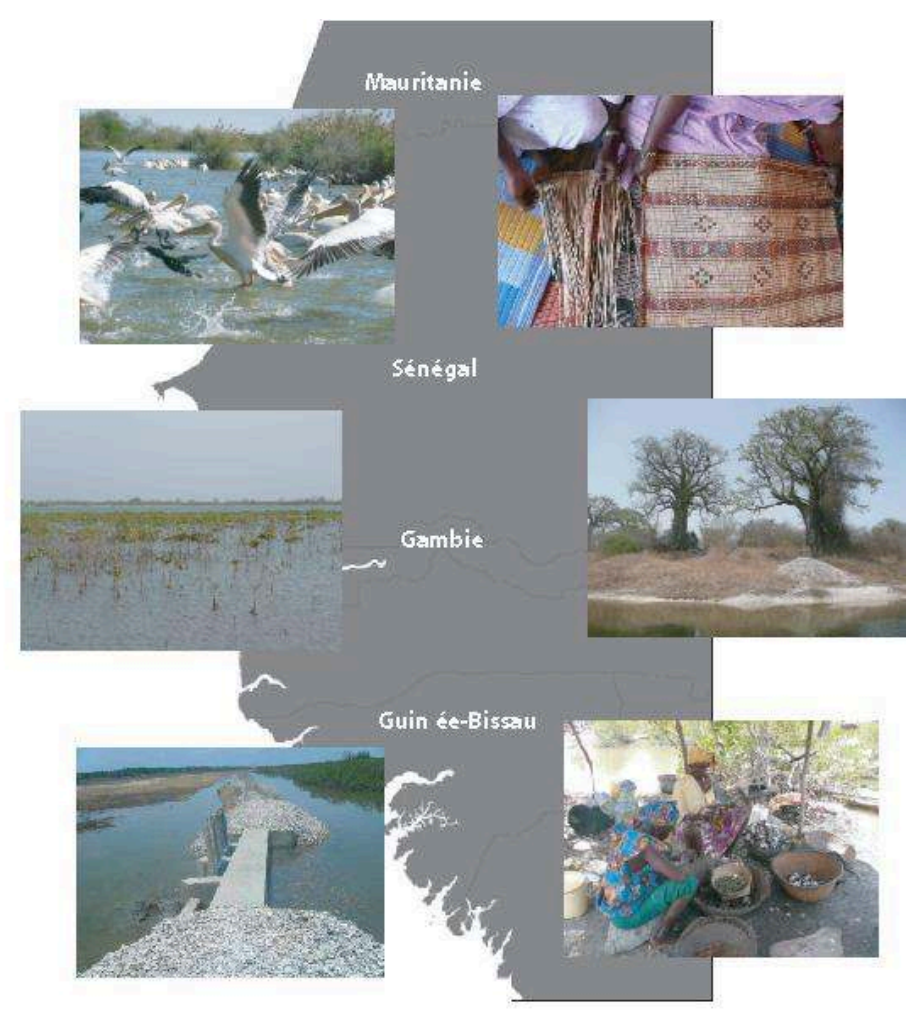

Source : PATEO, 2012

3 En s'appuyant sur les résultats obtenus dans le cadre du consortium PATEO, ce travail analyse la vulnérabilité et la résilience des socio-écosystèmes littoraux d'Afrique de l'Ouest (fleuve Sénégal, Saloum-Niumi, Casamance-Geba) en termes d'état de connaissances actuelles et s'interroge sur leur devenir.

\section{Matériels et méthode}

4 L'outil documentaire, notamment la très importante bibliographie existant sur le littoral ouest-africain, a été mobilisé afin de mieux documenter les questions environnementales, les activités humaines, les pratiques et les politiques publiques qui y sont mises en œuvre. Parallèlement, plusieurs méthodes ont été utilisées par les membres du laboratoire dans les différentes études. Il s'agit, entre autres, des méthodes de classifications automatiques (Soumaré, 2018; Diéye et al., 2021; Andrieu, 2021), de la photo-interprétation (GRDR et al., 2017; Barry, 2017; Diop, 2017 ; Sané, 2017; Thior et al., 2019) et de la cartographie participative (Cormier-Salem, Sané, 2017b ; Ehemba et al., 2017 ; Sané et al., 2017). Ces méthodes, qui font appel à l'utilisation de l'imagerie satellitaire et aérienne, ont abouti à une cartographie sur plusieurs dates et à différentes échelles, les unes portant sur l'évolution des mangroves (1972, 1979, 1986, 1990, 1999, 2000, 2005, 2010, 2016, et 2018), les autres sur l'évolution du trait de côte $(1968,1986,2004$ et 2017) et des terroirs rizicoles villageois (1968 et 2017).

5 À ces méthodes s'ajoutent l'analyse de la pluviométrie, des températures et du niveau moyen de l'océan (Sané, 2017 ; Sané et al., 2018 ; Descroix, 2018), les mesures de salinité 
des bolons et des nappes (Sané, 2017). Ces méthodes ont été couplées avec la collecte de données sur le terrain à l'aide de transects, de profils historiques, etc.

\section{Résultats}

\section{Dynamiques et recompositions des paysages littoraux en zone sénégalo-bissau-guinéenne}

6 L'analyse de la dynamique des paysages littoraux porte sur l'évolution des conditions climatiques, l'état de la mangrove, les mutations des terroirs rizicoles et des conditions de mises en valeur des parcelles rizicoles. En effet, plusieurs études du LMI PATEO ont été consacrées à ces questions (Descroix et al., 2016 ; Cormier-Salem et al., 2017a ; Niang, 2017 ; GRDR et al., 2017 ; Sané, 2017 ; Diop, 2018 ; Descroix, 2018 ; Soumaré, 2018 ; Thior, 2020 ; Diéye et al., 2021; Andrieu, 2021). Ces travaux montrent que les dynamiques qui s'observent dans ces milieux relèvent à la fois des forces externes (climat, érosion côtière, salinité et acidité des terres agricoles) et internes (pratiques sociales et politiques publiques) qui font évoluer activement ces socio-écosystèmes littoraux depuis le début des années 1970.

\section{Le climat, facteur déclenchant des dynamiques littorales}

7 Le climat est un élément déterminant dans l'évolution des milieux et des sociétés côtières ouest-africaines. Sa forte variabilité spatio-temporelle s'est traduite, surtout depuis la fin des années 1960, par une succession d'années sèches et d'années humides corrélée à une élévation remarquable des températures au cours de ces dernières décennies (Sané, 2017 ; Sané et al., 2018).

En ce qui concerne les précipitations annuelles, l'analyse de la figure 2 laisse apparaître quatre périodes majeures, caractéristiques de l'évolution des conditions climatiques en région ouest-africaine depuis le début $\mathrm{du} \mathrm{XX}^{\mathrm{e}}$ siècle. La période 1900-1950 est caractérisée par une pluviométrie relativement normale avec cependant quelques séquences sèches (1910-1913, 1942-1944 et 1948-1950). Cette période est suivie par une séquence très humide (1951-1967) matérialisée par des anomalies positives. À l'opposé de ces deux premières périodes à la pluviométrie normale à très excédentaire, la période 1968-1998 se démarque par l'absence quasi totale d'années à pluviométrie normale. Il s'agit d'une longue période durant laquelle les déficits pluviométriques ont été les plus intenses, leur variabilité spatiale forte et leurs conséquences sur les socioécosystèmes les plus prononcées. En effet, cette période de profonds déficits pluviométriques a été désastreuse pour les sociétés rurales du littoral ouest-africain, puisqu'elle a été le déclencheur des mutations socio-spatiales importantes observées dans cette zone, aggravant du coup la vulnérabilité de ces socio-écosystèmes (Sultan et al., 2015 ; Sané, 2017 ; Descroix, 2018 ; Sané et al., 2018). La quatrième période, 1999-2015, est marquée par l'importance des anomalies positives, comparativement à la période de sécheresse, avec cependant une nette alternance entre années excédentaires et années déficitaires. Cette période révèle donc une nette amélioration de la pluviométrie. Le retour de la pluviométrie à des conditions meilleures participe au regain de vitalité des écosystèmes même si les conditions socio-économiques restent 
encore fortement marquées par les profonds déficits de la période de sécheresse (1968-1998).

Figure 2. Variabilité des écarts à la moyenne des précipitations en zone soudano-sahélienne de 1900 à 2015.

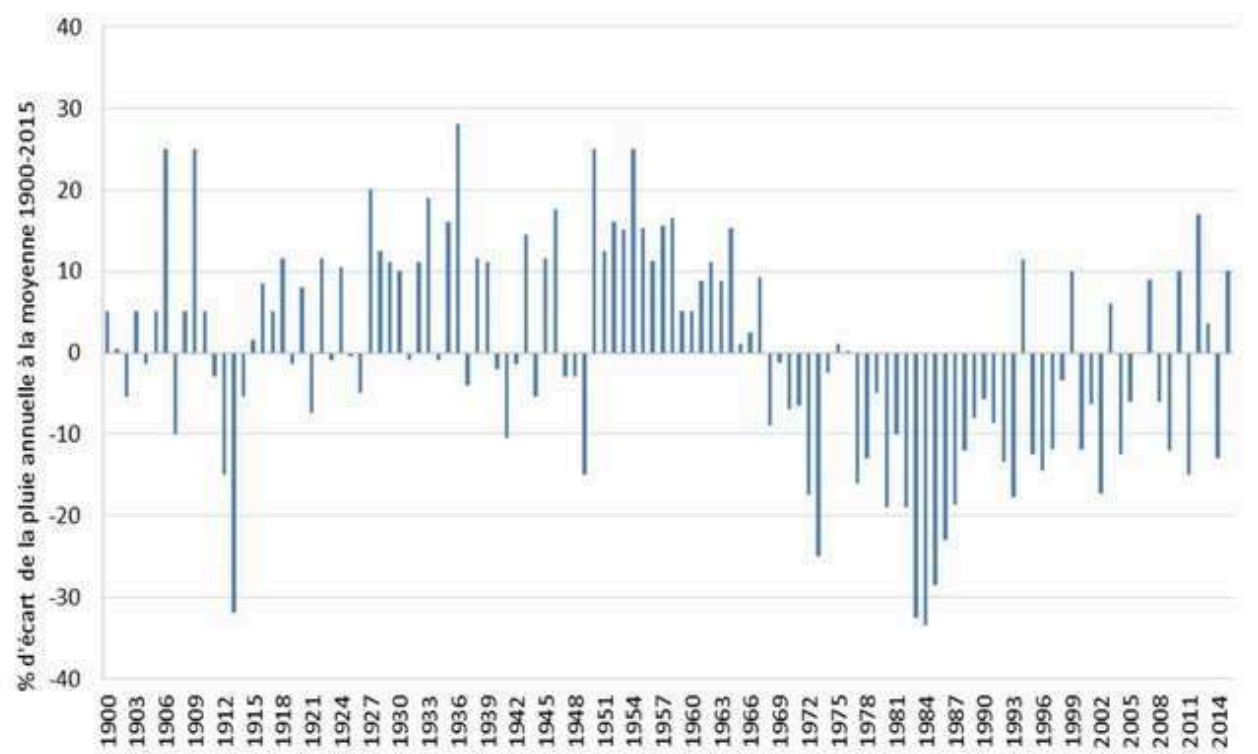

Source : Descroix, 2018

9 Au-delà de la pluviométrie, considérée comme le facteur qui influence le plus les activités humaines, d'autres paramètres climatiques comme la température sont aussi importants à prendre en considération dans l'analyse des conditions climatiques en zone côtière. La précarité actuelle des activités humaines, notamment agricoles, est aussi la conséquence de la hausse des températures (Sané, 2017). Les figures 3 et 4 montrent une importante augmentation des températures à Ziguinchor, ville située en zone climatique sud-soudanienne, donc à cheval entre les domaines climatiques nordsoudanien et sub-guinéen. On y remarque une hausse des températures minimales survenue depuis 1982 et celles maximales à partir de 1994. Cette hausse des températures, observable presque partout en Afrique de l'Ouest (Guichard et al., 2009 ; Sultan et al., 2015) est intervenue au moment où les précipitations étaient dans une situation de profond déficit. Cette hausse des températures minimales et maximales confirme la tendance au réchauffement observée à l'échelle mondiale. 
Figure 3. Évolution des anomalies de températures minimales à Ziguinchor de 1951 à 2014.

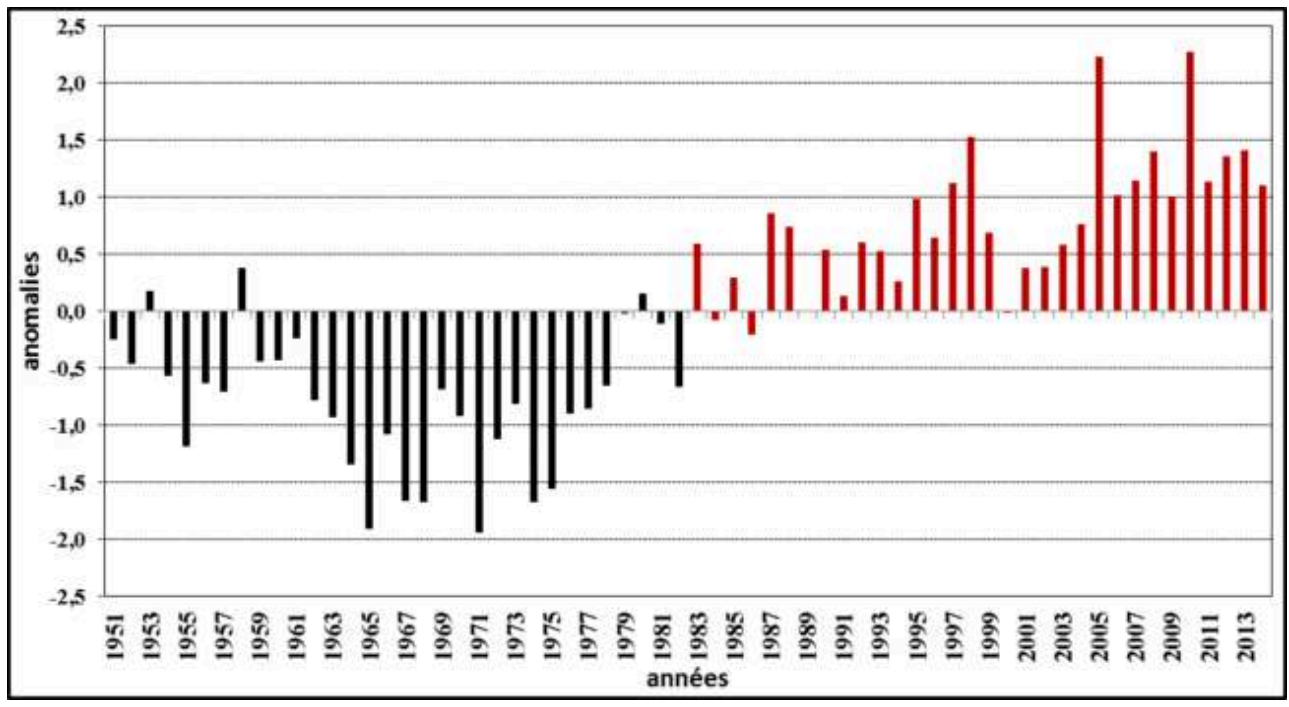

Source : Sané, 2017

Figure 4. Évolution des anomalies des températures maximales à Ziguinchor entre 1951-2014.

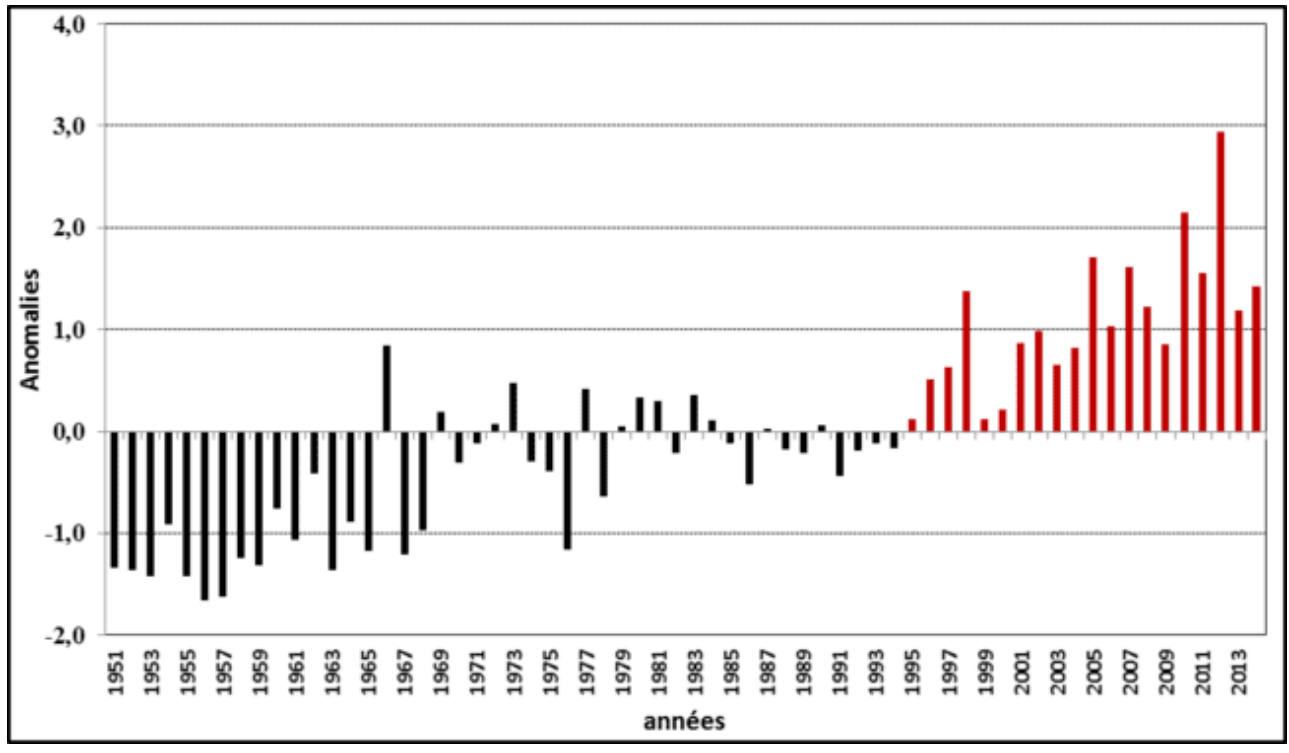

Source : Sané, 2017

10 Finalement, l'importance de la variabilité des conditions climatiques, particulièrement la pluviométrie et la température en zone littorale ouest-africaine, a entraîné des répercussions sur la dynamique des écosystèmes et des systèmes productifs.

La dynamique des paysages littoraux sénégalo-bissau-guinéens à travers l'exemple de la mangrove

11 Les nombreuses études réalisées par les membres du PATEO sur l'écosystème de mangrove du fleuve Sénégal, Saloum-Niumi, Casamance-Geba (Sané, 2017 ; Solly et al., 2018 ; Soumaré, 2018 ; Andrieu, 2021 ; Diéye et al., 2021) ont démontré l'importance des mutations qui y sont intervenues. En effet, à travers l'imagerie géospatiale, trois 
périodes majeures ont souvent été identifiées dans l'évolution de la mangrove. Il s'agit de la période des années 1950-1960, de la période 1968 à 1997, et de la période 1998 à nos jours. La première période, humide du point de vue pluviométrique, est marquée par une stabilité de la mangrove. La seconde période est marquée par une régression importante des surfaces de mangrove. Durant cette période, la mangrove a été éprouvée par les effets de la sécheresse, notamment à cause de l'hypersalinité des eaux et des sols mais aussi des actions de l'homme à travers la coupe du bois pour diverses raisons. La dernière période est marquée par une régénération importante des surfaces en lien avec le retour à de meilleures conditions pluviométriques et une prise de conscience des populations quant à l'importance de cet écosystème à travers les actions de reboisement et de revalorisation.

À titre illustratif, Soumaré (2018) a montré que la superficie de la mangrove avait fortement régressé dans la commune de Kafountine (Basse-Casamance), puisqu'elle est passée de 13 450,35 ha en 1972-1986 et à 1 280,26 ha en 1986-2000 (fig. 5). Par contre, entre 2000 et 2016, cette mangrove a connu une régénération (naturelle ou assistée) jusqu'à occuper une surface de 5897,41 ha.

Figure 5. Carte de la dynamique de la mangrove dans la Commune de Kafountine sur les périodes 1972-1986 et 2000-2016.

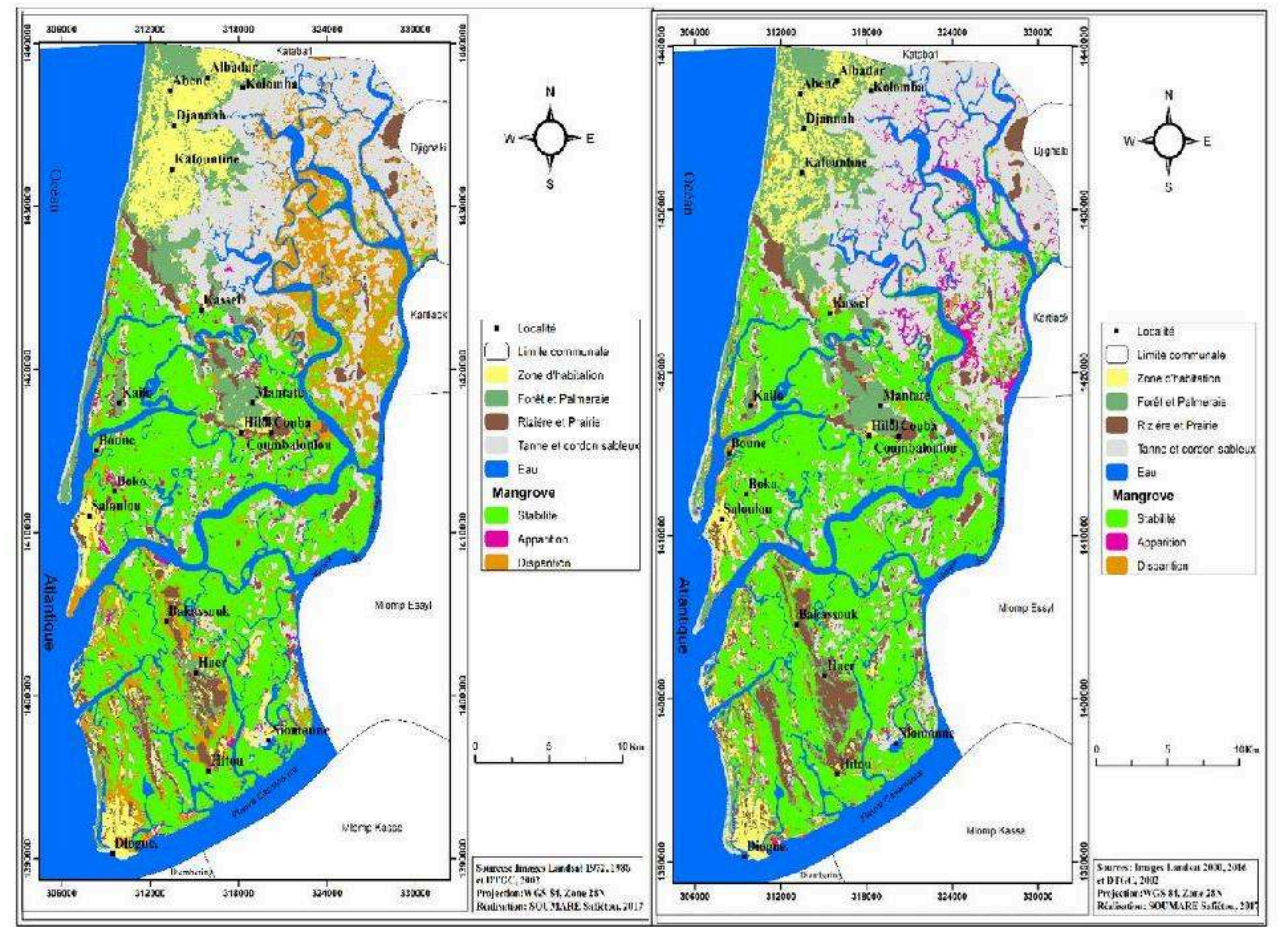

Source : Soumaré, 2017

Dans le Delta du Saloum, Andrieu (2021) a noté que la superficie de la mangrove est passée de $690 \mathrm{~km}^{2}$ en 1979 à $572 \mathrm{~km}^{2}$ en 1988 et $631 \mathrm{~km}^{2}$ en 2015. Du Delta du Saloum au Rio Cacine, Diéye et al., (2021) ont noté une régression de 23777 ha de mangrove dense entre 1971-1988, et de 104199 ha pour celle moins dense, une régénération bien visible dès 2000 et plus importante en 2010 avec l'augmentation de la mangrove dense (42 743 ha). 
Il est évident que la sécheresse des années 1970 à 1990, combinée aux différentes évolutions sociologiques et économiques, a favorisé l'évolution des pratiques agrosylvo-pastorales qui, à leur tour, ont largement contribué à d'importantes transformations des paysages littoraux. Cependant, le retour de la pluviométrie à une situation quasi normale est en train de redonner un regain de vitalité aux écosystèmes dont la régénération partiellement naturelle constitue un témoin essentiel. Les changements des espaces littoraux ouest-africains résultent également du relèvement du niveau de la mer dont une des expressions est l'envahissement des rizières par l'eau de mer et l'érosion côtière très active (Thior, 2020), aux conséquences socioenvironnementales relativement importantes.

Les mutations dans les paysages agraires littoraux d'Afrique de l'Ouest : cas de la riziculture

La frange littorale est considérée comme la zone la plus riche en rizières de toute l'Afrique occidentale (Portères, 1962; Cormier-Salem, 1992, 1994). Dans la zone d'intervention du PATEO, on retrouve les peuples riziculteurs comme les Diola, Baïnounk, les Manjacque, les Balante, entre autres. La principale caractéristique de ces populations est la pratique de la riziculture inondée. Ce système, à la fois écologique et social, trouve son expression la plus originale dans la riziculture de mangrove. Les mangroves endiguées, défrichées et dessalées sont converties en rizières au prix d'un travail accumulé sur plusieurs générations (Pélissier, 1966 ; Cormier-Salem, 1994 ; Sané, 2017 ; Sané et al., 2018).

L'état des lieux de l'évolution de la riziculture en zone littorale ouest-africaine issue des travaux du LMI PATEO (Sané, 2017 ; GRDR, 2017 ; Diop, 2017 ; Sané et al., 2018 ; Mendy, 2018) révèle que la riziculture est confrontée aux problèmes de salinisation, d'acidification et d'ensablement des terres. Ces problèmes sont le résultat conjoint de l'altération pluviométrique des années 1970 à 1990 et du relâchement de l'entretien des digues et diguettes par les hommes du fait des départs en migration et d'autres facteurs, en particulier du désintérêt des jeunes pour les travaux liés à la riziculture, ces travaux étant basés essentiellement sur l'énergie humaine. Cette situation a provoqué des mutations dans les paysages agraires, particulièrement le recul de la riziculture, une activité ancestrale de haute importance aux plans alimentaire, cultuel et culturel. Dans le terroir rizicole villageois de Coubanao (Sénégal) et de Varela Yale en Guinée-Bissau les terres rizicoles ont diminué entre 1968 et 2015 (fig. 6 et 7). 
Figure 6. Carte de la dynamique du terroir rizicole villageois de Coubanao (Sénégal) en 1969 et 2015.

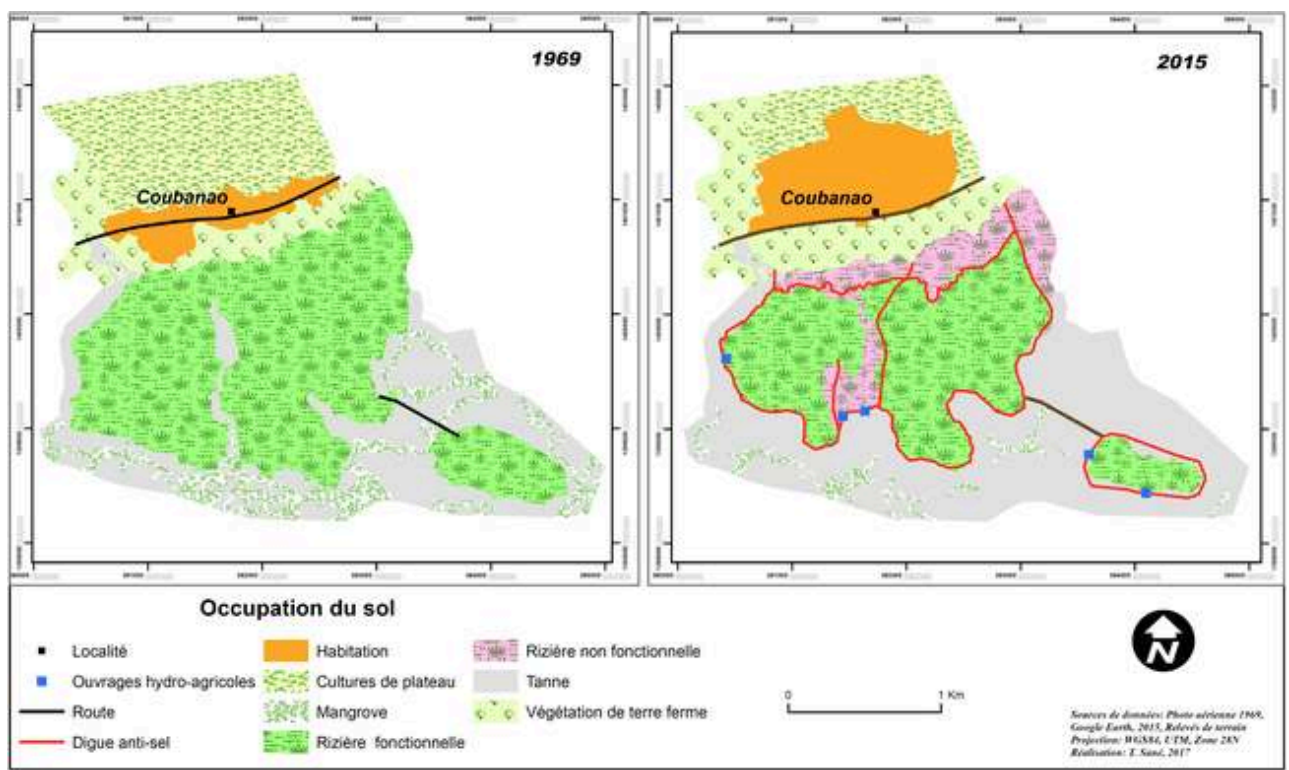

Source : Sané, 2017

Figure 7. Carte de la dynamique du terroir rizicole villageois de Varela Yale (Guinée-Bissau) en 1968 et 2015.

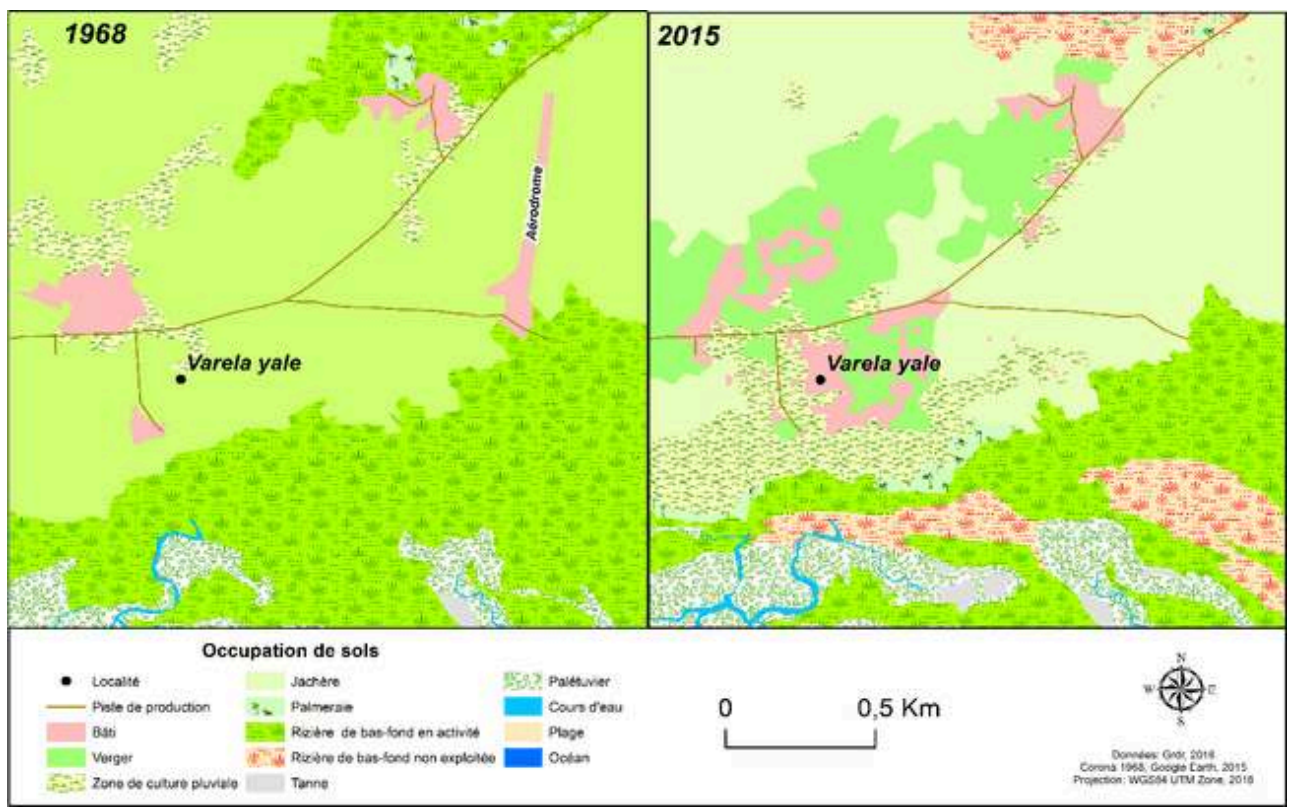

Source : GRDR et al., 2017

La cartographie participative (Cormier-Salem et al., 2017 ; Ehemba et al., 2017 ; Sané et al., 2017) a permis d'appréhender la perception des populations sur la dynamique en cours dans leurs terroirs (fig. 8). L'analyse de la figure 8 (situation d'il y a cinquante ans et situation actuelle) montre une forte recomposition des unités paysagères du terroir villageois de Diembering. Ce résultat révèle une bonne connaissance des populations paysannes sur la recomposition de leurs terroirs dans un contexte de changements globaux. 
Figure 8. Carte de la dynamique des unités paysagères dans le terroir villageois de Diembering révélé par la population locale : il y a plus de 50 ans (à gauche) et terroir actuel (à droite).

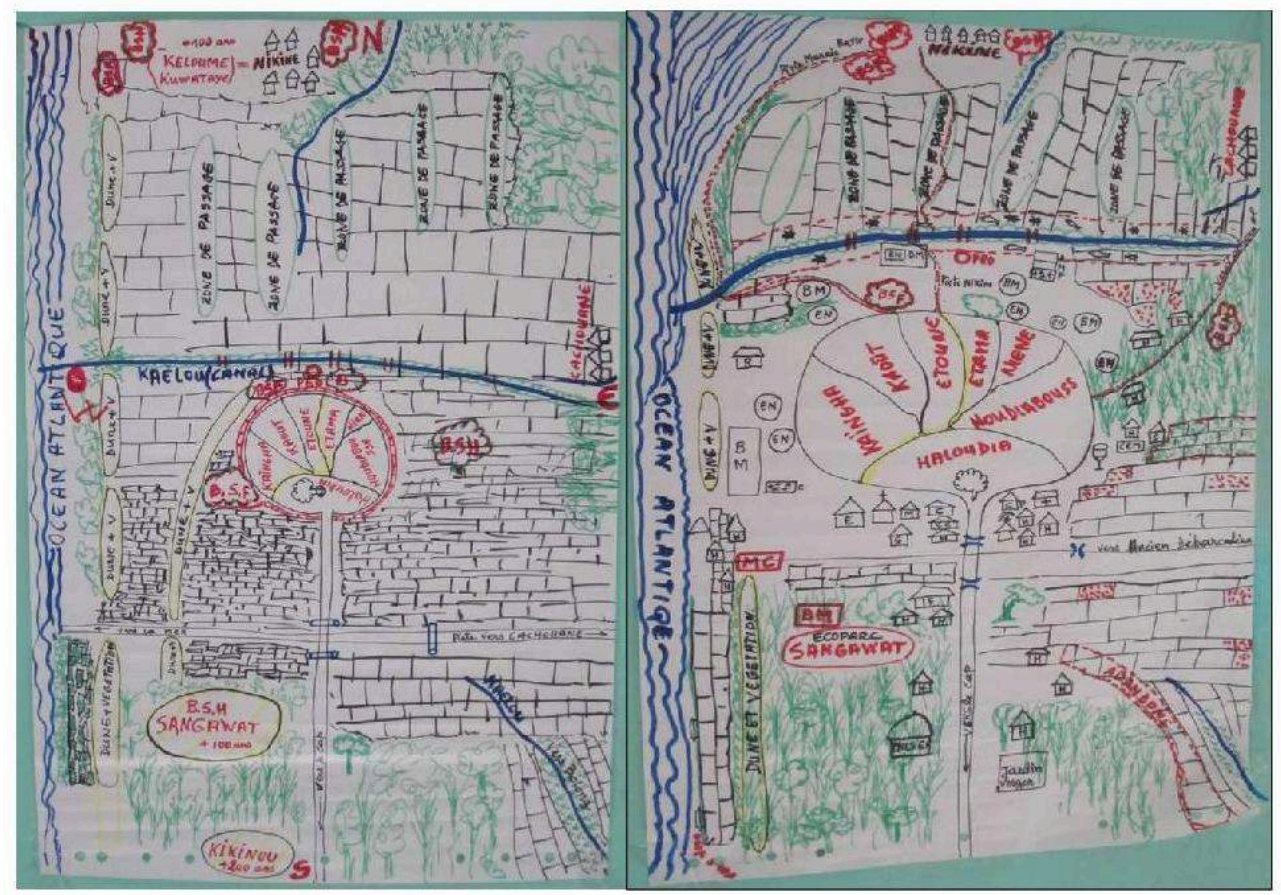

Source : Sané, 2017

18 De manière générale, la dynamique de la mangrove et la recomposition des terroirs rizicoles constituent des indicateurs majeurs de la vulnérabilité des socio-écosystèmes littoraux en Afrique de l'Ouest. Malheureusement, elles ne sont pas les seuls facteurs qui contribuent à l'évolution des zones littorales. L'érosion côtière, très active dans les secteurs sableux de ce littoral, contribue fortement à la fragilisation de l'environnement côtier et des systèmes productifs et socio-économiques.

\section{L'érosion côtière, un facteur très dynamique en région littorale ouest-africaine}

Cette partie examine l'état actuel de l'érosion côtière, phénomène très dynamique le long de la frange littorale sénégalo-bissau-guinéenne (Ba, 2013; Barry, 2017 ; Thior et al., 2019; Thior, 2020). Les résultats issus des travaux du PATEO et d'autres auteurs comme Faye (2010) ont montré que l'intensité de l'érosion sur les littoraux sénégalobissau-guinéens résulte à la fois de l'orientation et de l'importance des courants, de la fréquence des vents océaniques et du relèvement du niveau de la mer (fig. 9) en rapport avec le changement climatique (Descroix, 2018 ; Thior, 2020). 
Figure 9. Évolution rapide de la hausse du niveau moyen global de l'océan en Afrique de l'Ouest.

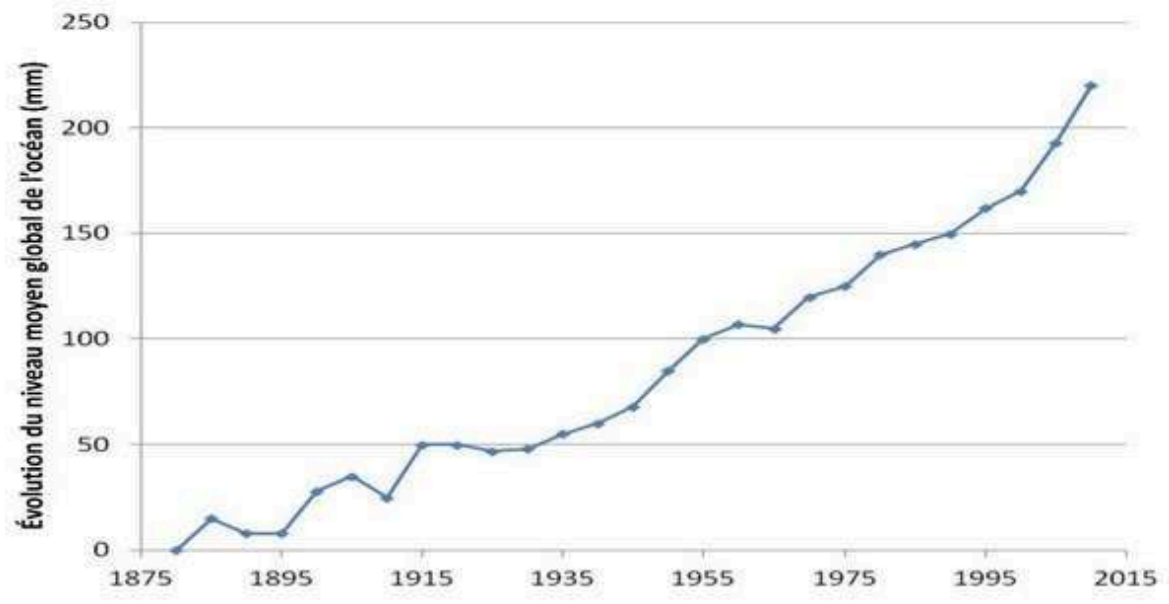

Source : Descroix, 2018

20 L'érosion est très active dans bien des secteurs du littoral ouest-africain. Les conséquences de ce dynamisme érosif sont très importantes sur le littoral sénégalais. Trois cas illustrent bien l'agressivité érosive au niveau des côtes sableuses sénégalaises. Il s'agit de la rupture volontaire de la flèche sableuse de la langue de Barbarie dans le delta du fleuve Sénégal (fig. 10), de celle de la flèche de Sangomar dans le delta du Saloum (fig. 11) et de l'évolution du trait de côte en Basse-Casamance (fig. 12).

Figure 10. Carte de la flèche sableuse de la langue de Barbarie au Sénégal avant et après sa rupture volontaire survenue en septembre 2003.

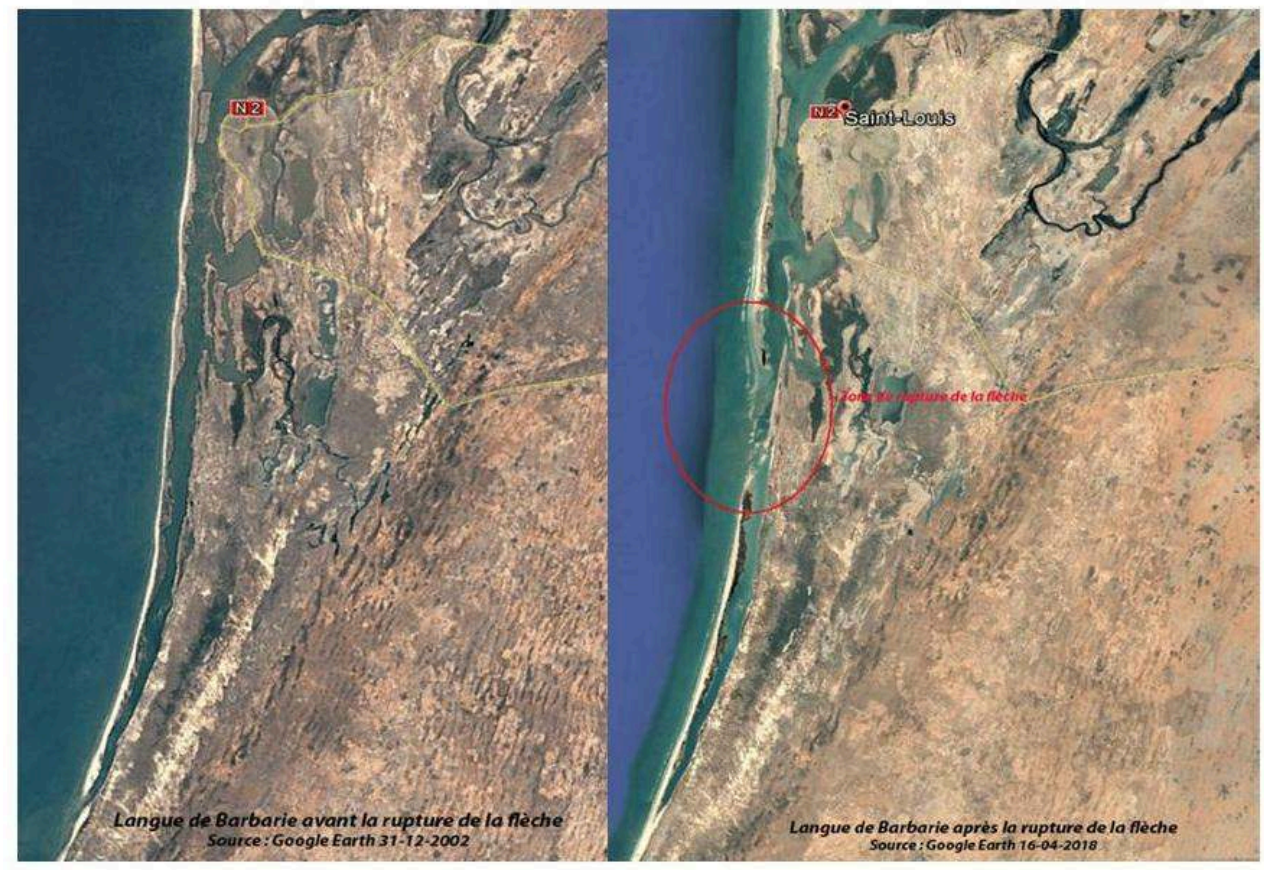

Source : Google Earth 2002 et 2018 
Figure 11. Carte d'évolution de la flèche de Sangomar entre 1972 et 2005 dans le delta du Saloum.

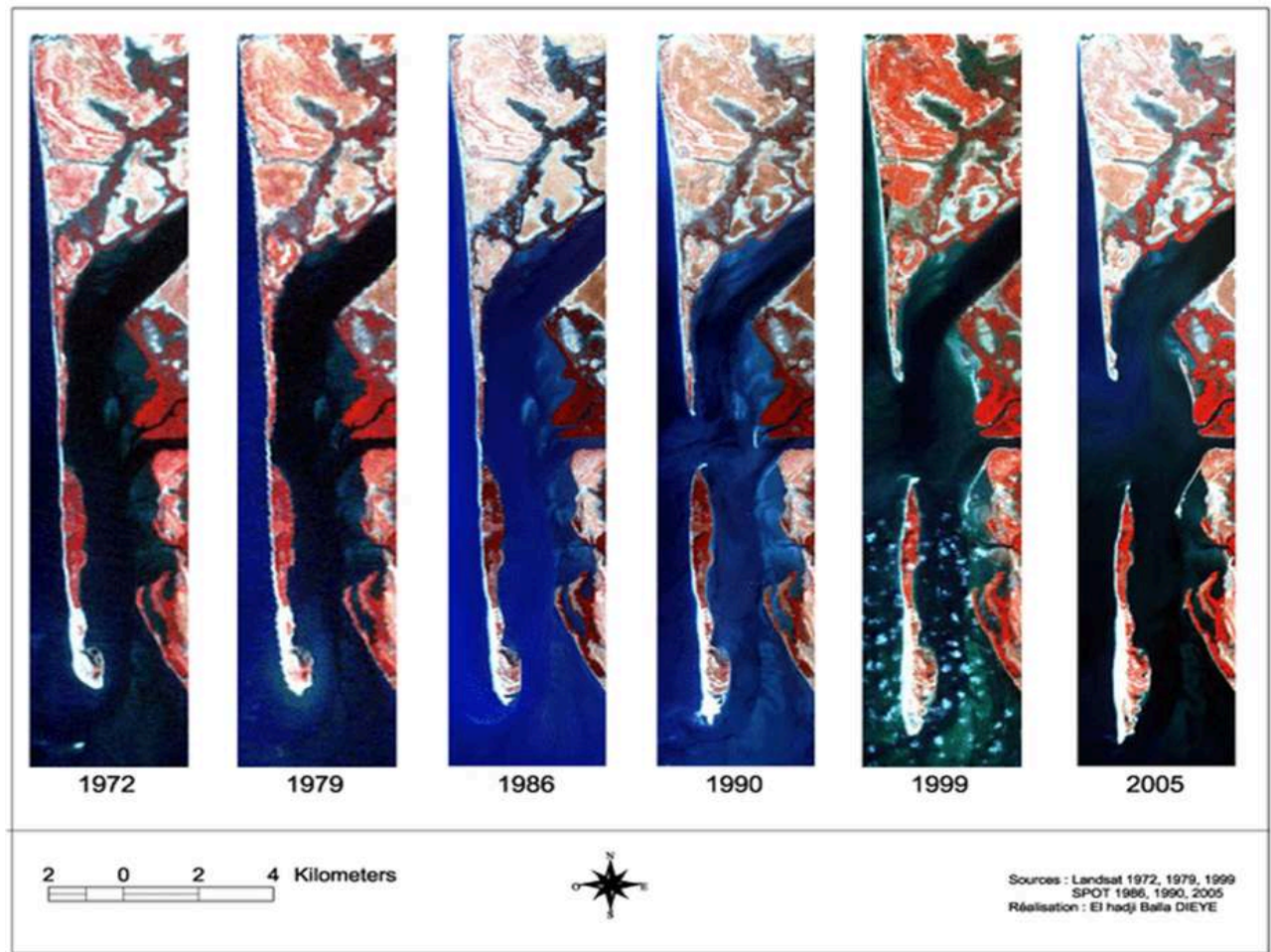

Source : Diéye, 2007

Figure 12. Carte d'évolution du trait de côte dans la zone de Diogué en Basse-Casamance entre 1968 et 2017.

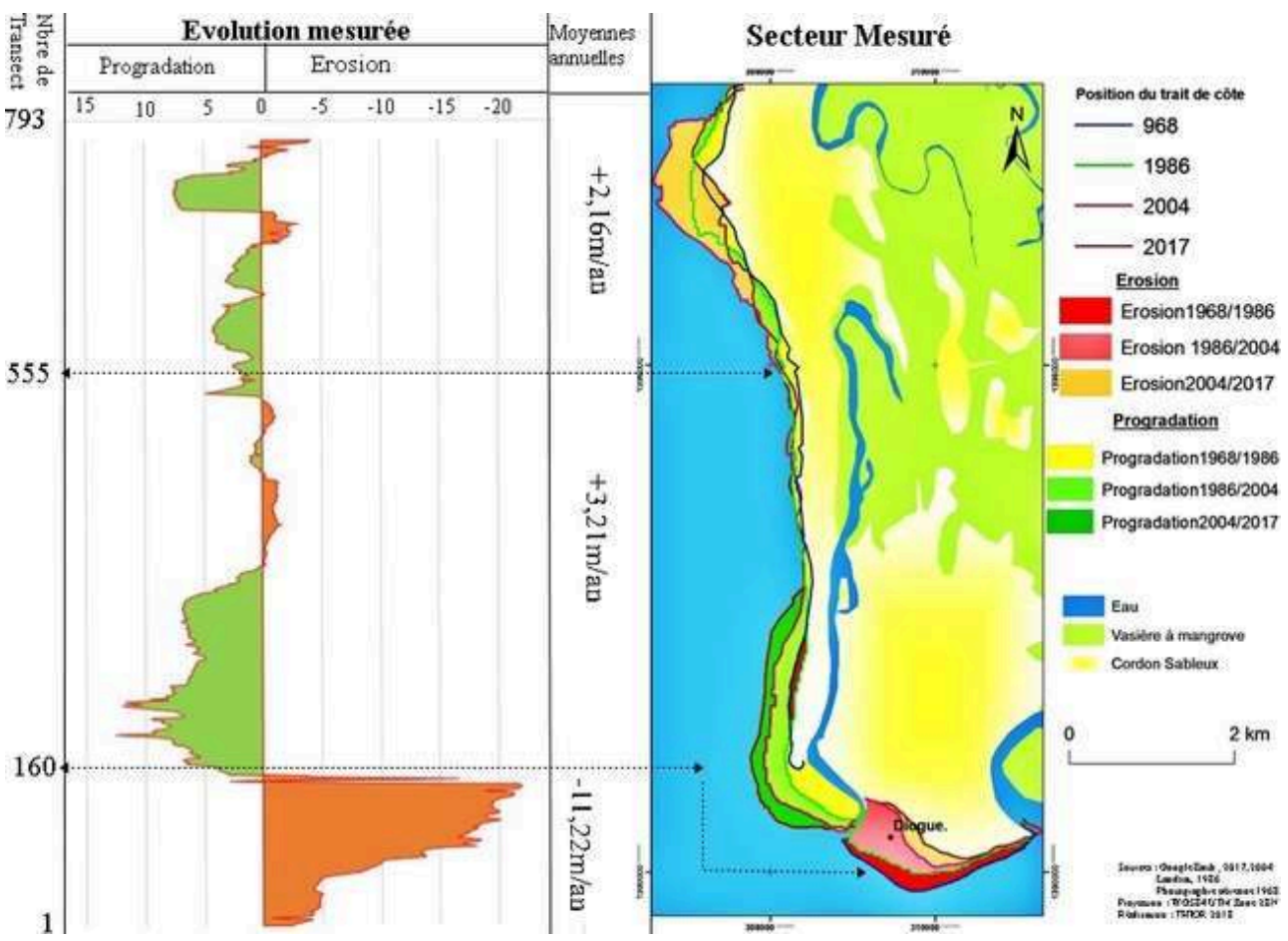

Source : Thior, 2020 
21 résulte à la fois de facteurs naturels (élévation du niveau de la mer, importar
direction des houles et des courants marins, etc.) et anthropiques (aména
divers).
La résilience des socio-écosystèmes littoraux : des pratiques
paysannes aux politiques publiques

Devant l'adversité induite par la conjonction de diverses forces (internes et externes), les sociétés rurales des littoraux ouest-africains ont toujours tenté de s'adapter aux changements et contraintes de leurs écosystèmes, notamment dans leurs composantes environnementale, sociale et économique. Plusieurs exemples peuvent être considérés comme des cas de résilience de la population locale par rapport aux changements globaux. Cette partie traite de la capacité de résilience de ces socio-écosystèmes côtiers du littoral sénégalo-bissau-guinéen.

Les pratiques paysannes, éléments de résilience face aux contraintes naturelles ?

Les sociétés rurales des riziculteurs d'Afrique de l'Ouest comme les Diola de BasseCasamance (Sénégal) se sont toujours adaptées au milieu très contraignant dans lequel elles évoluent. Elles ont organisé leurs activités rizicoles en fonction des différentes unités géomorphologiques, où digues et diguettes délimitent les propriétés foncières, mais servent également à la gestion efficiente des eaux (Cormier-Salem, 1999; Sané et al., 2018; Mendy, 2018). Autrement dit, les techniques agricoles mises en œuvre sont fonction des milieux et des possibilités qui leur sont offertes. Le rôle de la toposéquence est fondamental dans la déclinaison des opérations culturales et des plantes cultivées (Sané, 2017). L'espace rizicole, en zone littorale sénégalo-bissau-guinéenne, est matérialisé par trois unités géomorphologiques sur lesquelles sont portés les aménagements agricoles (plateau, versant et bas-fond). L'adaptation à une telle configuration permet de retrouver des rizières sous les palmeraies, sur les versants, dans les dépressions des plateaux, dans les plaines des vallées drainées par l'eau douce et sur les langues de sable de l'estuaire de la Casamance. Une telle stratégie dans les opérations culturales rizicoles témoigne d'une résilience de ces populations du littoral ouest-africain par rapport aux contraintes naturelles.

Par ailleurs, le retour de la pluviométrie à une situation plus satisfaisante que celle des années de sécheresse donne de l'espoir aux populations paysannes qui ont su, même en période de sécheresse, développer des stratégies (développement de la riziculture de plateau, adoption de variétés de riz à cycle court, etc.) pour maintenir la riziculture. Les organisations paysannes (entente de Diouloulou, KADES des Kalounayes, etc.) et la jeunesse déploient des efforts pour redynamiser l'activité agricole (fig.13). La population locale s'emploie également à la diversification des activités rémunératrices comme le développement des plantations (vergers et champs d'anacardiers, petit commerce, etc.) et les jeunes surtout répondent par la mobilité. L'introduction, quoique timide, des motoculteurs en Basse-Casamance constitue également une innovation de taille dans les opérations culturales qui, naguère ne se faisaient qu'avec des outils manuels comme le Kajendu ${ }^{1}$. Ces comportements innovants peuvent alors être 
considérés comme des éléments de résilience des socio-écosystèmes littoraux ouestafricains.

Figure 13. Repiquage des plants de riz par les jeunes de Marougoune, village de Basse-Casamance.

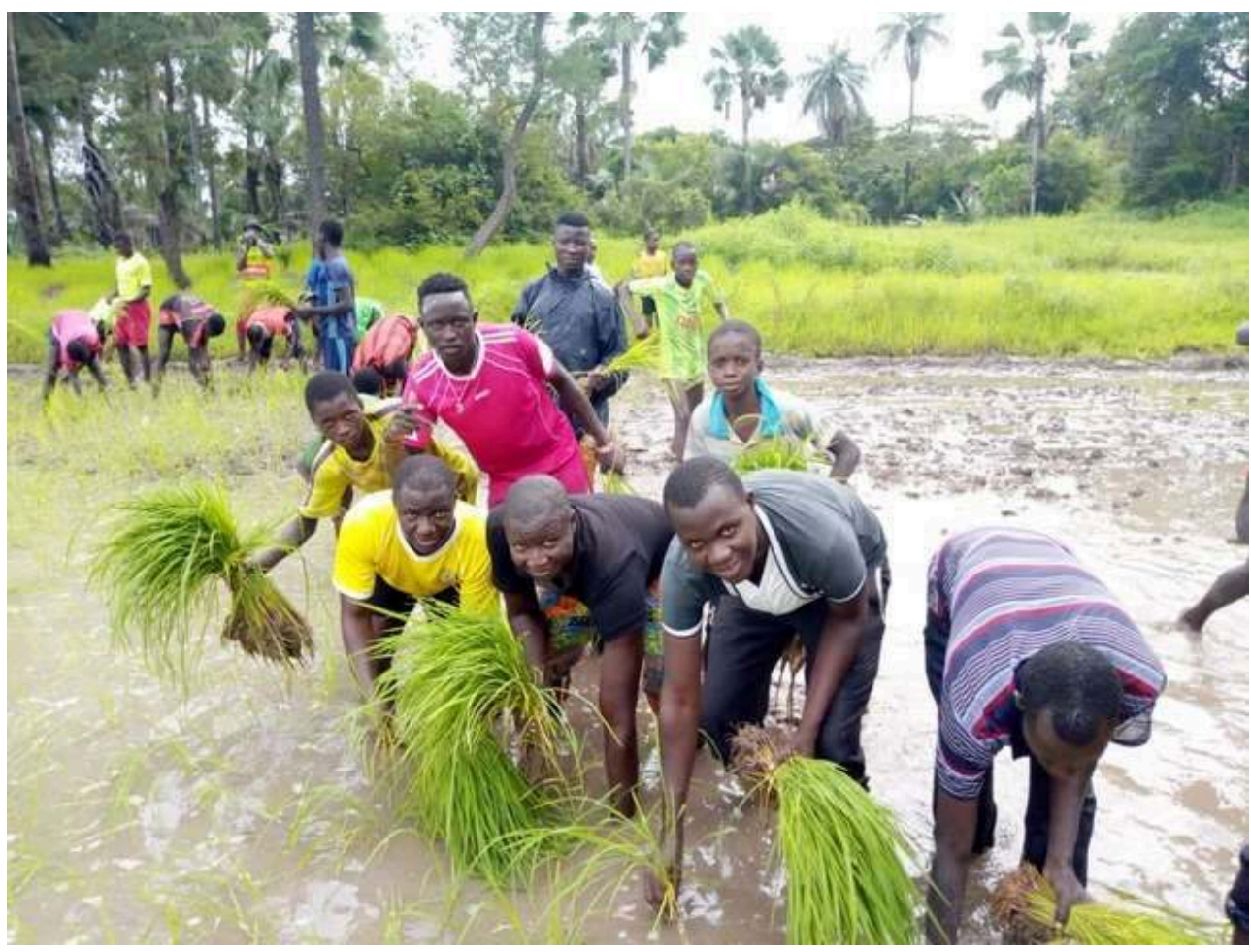

Crédit : T. Sané, septembre 2018

Des politiques publiques parfois inadaptées aux réalités des socio-écosystèmes ?

Pour faire face à de nombreuses contraintes imposées par les forces externes (sécheresse des années 1970 à 1990) et la volonté politique de développer l'agriculture, la plupart des États ouest-africains avaient mis en place, dans les socio-écosystèmes littoraux, d'importants programmes et projets d'aménagements hydro-agricoles à l'image des barrages anti-sel et digues de retenue des eaux en Basse-Casamance (fig. 14). C'est le cas du Programme agricole (1960-1980) avec un encadrement très rapproché des agriculteurs et la Nouvelle Politique Agricole (NPA) au début des années 1980, qui consacra le désengagement de l'État vis-à-vis des activités agricoles. Les politiques publiques agricoles, notamment dans le domaine rizicole, ont eu des résultats mitigés. Si certains aménagements hydro-agricoles en Basse-Casamance ont contribué au maintien de la riziculture avec la mise en place des programmes et projets de développement rizicole (SOMIVAC, PIDAC, PADERCA, PPDC, etc.) structurés autour de l'accompagnement des paysans et de la réalisation des digues de protection des rizières, d'autres cependant ont été adaptés aux réalités des socio-écosystèmes littoraux. Ces réalités ont mis à mal, dans la plupart des cas, la résilience de ces socioécosystèmes fragiles. En effet, certains ouvrages hydroagricoles à l'image du barrage d'Affiniam (aménagements secondaires non réalisés) ont renforcé la fragilité et la vulnérabilité de ces écosystèmes du fait de l'absence, dans certains cas, des études d'impacts environnementaux et de l'inadaptation de certaines réalisations aux réalités locales (destruction de certaines zones de mangrove censées être érigées en rizières 
avec un échec retentissant: cas des projets ILACO à Tobor et à Diéba près de Marsassoum.

Figure 14. Barrage anti-sel (à gauche : Affiniam, 2011) et digue anti-sel (à droite : Thionck-Essyl, 2015) en Basse-Casamance (Sénégal).

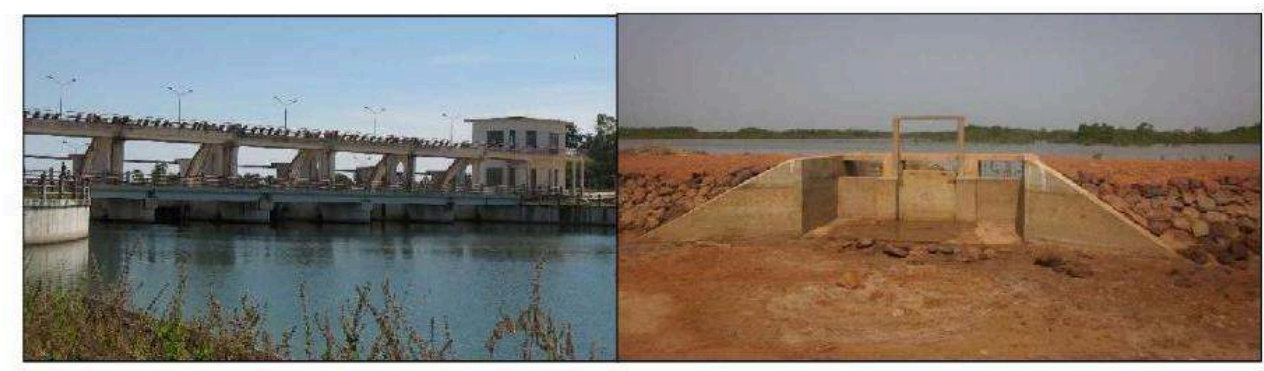

Crédit : T. Sané

Selon Sène (2015), les compétitions sur le littoral entre projets de territoire, à diverses échelles (du local à l'international), sont manifestes, et plus particulièrement les tensions entre, d'une part, les pêcheurs, exploitants et occupants traditionnels de cet espace, et d'autre part les acteurs extérieurs (pêcheurs industriels, touristes, spéculateurs immobiliers, entre autres). Cela est d'autant plus inquiétant que la zone côtière sénégalaise par exemple concentre environ $66,6 \%$ de la population nationale, $67,7 \%$ des actifs du secteur agricole sur environ $31 \%$ des superficies cultivées du pays et $72 \%$ des actifs du secteur industriel. Elle participe pour $67,9 \%$ à la réalisation du produit intérieur brut du Sénégal (MEPN/DEEC, 2007). Devant l'importance des enjeux environnementaux et de gouvernance sur les régions côtières, l'État du Sénégal en rapport avec les institutions régionales ou internationales a mis en place des mécanismes de protection des ressources littorales. La naissance du Programme Régional de Conservation des Aires Marines Protégées (PRCM) en 2004 (Bamboung, Joal-Fadiouth, Saint-Louis, Cayar et Abéné) et des aires communautaires (comme Mangagoulack, en Basse-Casamance) en sont une parfaite illustration (fig. 15). C'est en ce sens que Cormier-Salem (2018) rappelle que l'analyse des processus de patrimonialisation des mangroves (de leur sanctuarisation à leur conservation avec et pour les hommes) met en évidence l'impact des grandes rencontres internationales (Millenium Ecosystem Assessement en 2005; Objectifs du Développement Durable, 2015 , etc.). Celles-ci rythment l'agenda des politiques publiques, le poids des conventions internationales (comme la Convention sur la Biodiversité de 1992) et l'efficience de nouveaux mécanismes pour gérer durablement nos relations à l'environnement. Elles rythment aussi la diversité des réponses des acteurs locaux (savoirs, pratiques, logiques, stratégies) aux initiatives, publiques ou privées, de conservation de la biodiversité et de préservation des services écosystémiques (Cormier-Salem, 2019). 
Figure 15. Carte de la situation géographique des Aires Marines Protégées (AMP) au Sénégal.

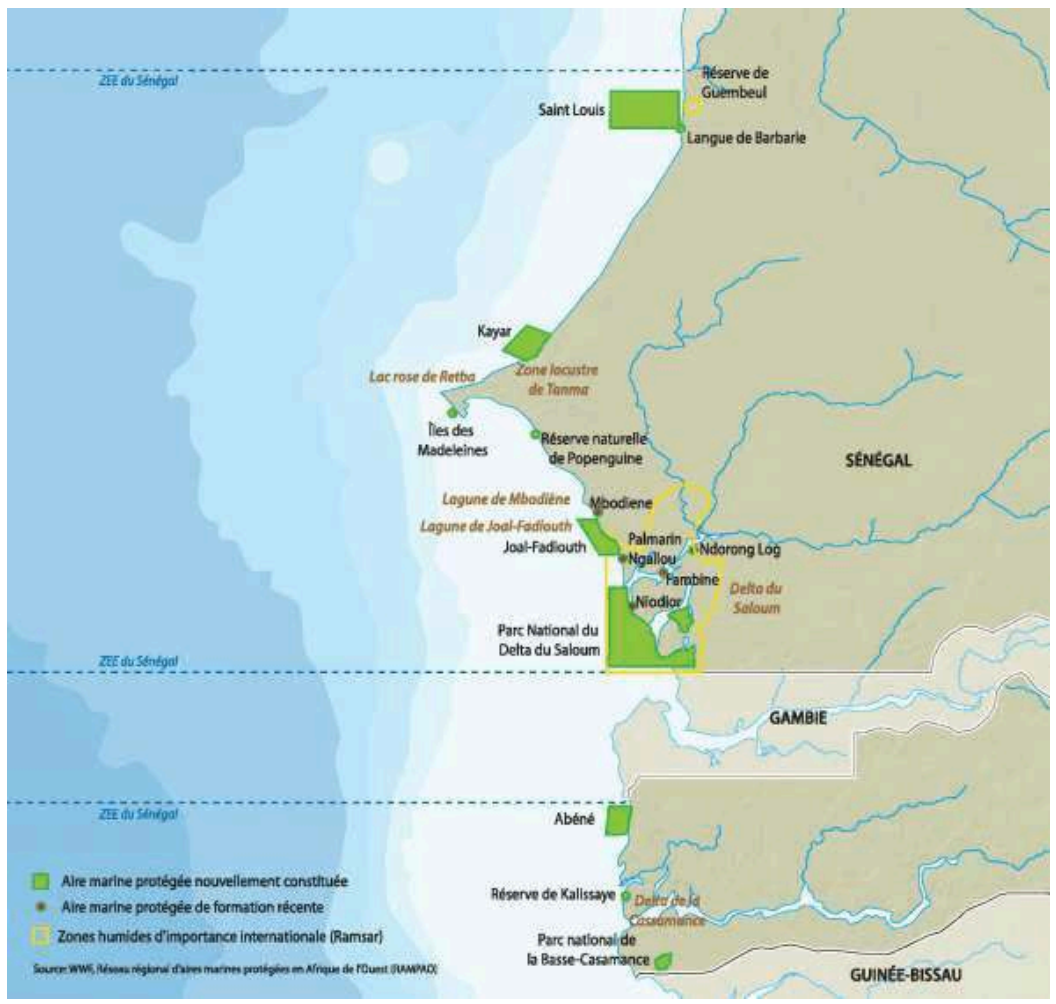

Source : DAMCP, 2012

27 La plupart des politiques publiques mises en œuvre sont souvent contradictoires avec la philosophie de préservation des ressources naturelles côtières. C'est le cas des accords de pêche avec l'Union européenne et les pays asiatiques, l'exploitation minière à l'image du zircon dans certaines zones du littoral sénégalo-bissau-guinéen (Niafrang en Basse-Casamance, Madina Varela en Guinée-Bissau), l'implantation d'usines d'huile et de farine de poisson à Abéné en Basse-Casamance. Ces initiatives sont néfastes pour l'environnement côtier ouest-africain qui continuera, si rien n'est fait, à subir les conséquences négatives des changements globaux. En effet, des effluents versés dans la nature (fig. 16) par l'usine de farine de poisson de l'entreprise chinoise à Abéné en Basse-Casamance posent un sérieux problème pour l'environnement et la santé humaine. 
Figure 16. Effluents versés dans la nature par l'usine de farine de poisson de l'entreprise chinoise à Abéné en Basse-Casamance.

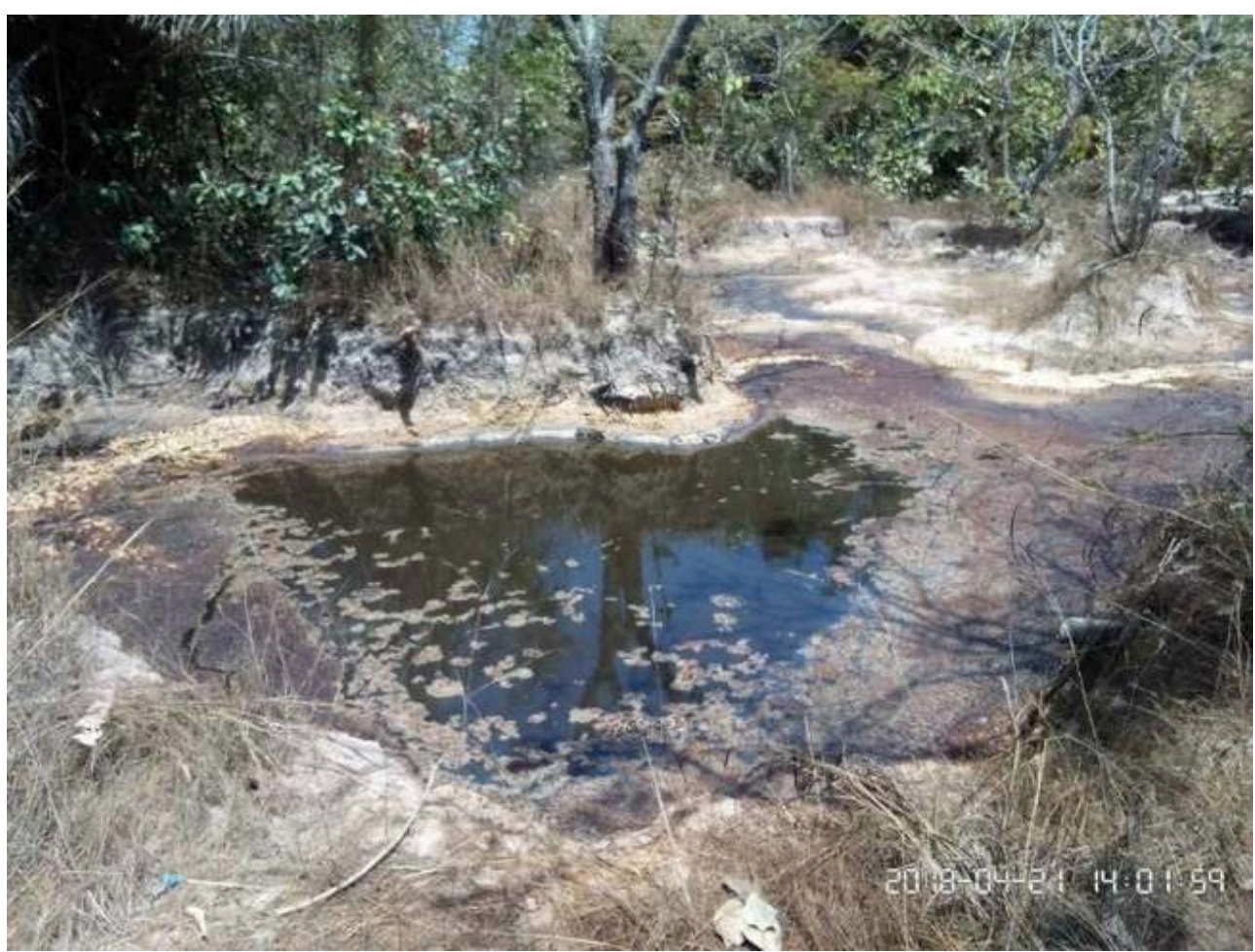

Crédit : T. Sané, avril 2019

\section{Conclusions}

Les socio-écosystèmes littoraux ouest-africains, bien que très riches sur le plan écologique et socio-économique, sont confrontés actuellement à de multiples contraintes qui provoquent ou renforcent leur vulnérabilité. Celle-ci est révélée, en partie, par la forte variabilité climatique, considérée comme un facteur déterminant à l'origine de la transformation de ces paysages littoraux.

Les mutations observées dans la mangrove en constituent un bon exemple. La dynamique de cet écosystème s'est manifestée par deux phases majeures. Il s'agit de la forte dégradation de la mangrove durant la sécheresse des années 1970 à 1990 et du regain de vitalité de cet écosystème à la faveur du retour actuel à la normale des précipitations et de la prise de conscience des communautés littorales sur son importance.

30 Le recul de la riziculture, induit à la fois par la salinité et par l'acidité des terres rizicoles, constitue une autre préoccupation des communautés paysannes des littoraux ouest-africains. La dégradation des terres rizicoles est aussi le résultat du relâchement de l'entretien des digues et diguettes par les hommes, qui cache une autre préoccupation, celle de la faiblesse du nombre de bras valides. Cependant, tenant compte des enjeux sociétaux et économiques de la culture du riz dans les sociétés littorales ouest-africaines, le maintien de cette activité s'exprime à travers le regard nouveau porté par les organisations paysannes et la jeunesse sur les activités rizicoles. 
31 L'intensité manifeste de l'érosion côtière, notamment dans les secteurs sableux de la frange littorale, constitue par ailleurs un élément majeur de la fragilisation des littoraux ouest-africains. La plupart des études sur ce sujet montrent l'urgente nécessité de procéder à des aménagements littoraux adéquats si l'on veut maintenir et renforcer la résilience de ces socio-écosystèmes face aux changements globaux de plus en plus manifestes et intenses.

Face à cette adversité, les sociétés rurales des littoraux ouest-africains et les États développent des stratégies d'adaptation, aussi partielles soient-elles, qui permettent leur maintien dans ces milieux vulnérables et fragiles. Cependant, les contradictions souvent relevées dans les politiques publiques ne militent pas forcément en faveur d'une résilience plus accrue de ces socio-écosystèmes littoraux dans un contexte de changement climatique redouté et redoutable.

\section{BIBLIOGRAPHIE}

ANDRIEU J. (2021), «L'évolution de la mangrove (1979-2019) du Saloum au Gêba, par télédétection ", Actes du Colloque international LMI-PATEO-UASZ, tenu à l'Université Assane Seck de Ziguinchor (Sénégal) du 19 au 22 novembre 2019, pp. 537-545.

BA K. (2013), Apport de la télédétection et des SIG dans l'étude de l'évolution de la langue de Barbarie et de l'estuaire du fleuve Sénégal, Thèse de doctorat, Université Gaston Berger de Saint-Louis (Sénégal), $218 \mathrm{p}$.

BADIANE L.M. (2016), Gestion hydrologique et spatiale d'un hydrosystème aménagé : le marigot de Bignona en Basse Casamance, Thèse, Université Gaston Berger de Saint Louis, 435 p.

BARRY M. (2017), Érosion côtière et impacts dans la commune de Kafountine (Basse-Casamance), Mémoire de master, Département de Géographie, Université Assane Seck de Ziguinchor, 123 p.

CORMIER-SALEM M.-C. (1992), Gestion et évolution des espaces aquatiques : la Casamance, Paris, Editions de l'ORSTOM, Collection Etudes et Thèses, 583 p.

CORMIER-SALEM M.-C. (1994), Dynamique et usages de la mangrove dans les pays des rivières du Sud (du Sénégal à la Sierra Leone), ORSTOM, collections Colloques et Séminaires, Paris, 357 p.

CORMIER-SALEM M.-C. (1999), Rivière du sud: Société et mangrove ouest africaine, vol. 1, Paris, édition IRD, $416 \mathrm{p}$.

CORMIER-SALEM M.-C. (2019), « Jeux d'échelles et solidarités territoriales : vers une gouvernance multiscalaire des aires protégées ", in BOULAY S., FANCHETTE S. (éd.), La question des échelles en sciences humaines et sociales, Versailles, Montpellier, Quæ, IRD, pp. 72-90.

CORMIER-SALEM M.-C., DESCROIX L. \& DIAKHATE M.M. (éd.) (2017a), « Sciences participatives et gouvernance des patrimoines et territoires des deltas ", Actes du colloque international du Laboratoire Mixte Internationale »Patrimoines et Territoires de l'Eau », 11-14 mai 2016, Université Gaston Berger de Saint-Louis du Sénégal, Paris, L'Harmattan, 370 p. 
CORMIER-SALEM M.-C., SANE T. (2017 b), « Définir un cadre méthodologique commun en cartographie participative ", Revue d'ethnoécologie, 11, pp. 1-18.

DESCROIX L. (2018), Processus et enjeux d'eau en Afrique de l'ouest soudano-sahélienne, Paris, EAC, $320 \mathrm{p}$.

DESCROIX L., DJIBA S., SANE T. \& TARCHIANI V. (éd.) (2016), « Eaux et sociétés face au changement climatique dans le bassin de la Casamance ", Actes de l'Atelier scientifique et du lancement de l'initiative "Casamance : un réseau scientifique au service du développement en Casamance" ", 15-17 juin 2015, Hôtel Kadiandoumagne de Ziguinchor, Sénégal, Paris, L'Harmattan, $243 \mathrm{p}$.

DESCROIX L., MENDY A., D'ALESSANDRO C., LAMBERT L., DIONGUE-NIANG A., DACOSTA H., SOW M., MARUT J.-C., DIALLO M., RUË O., SENGHOR M.-J. \& BOUAÏTA Y. (2018), « Politiques publiques ou réchauffement climatique sur le littoral ouest-africain : quelle résilience face à quels aléas ? ", Conférence IED/PRESA Changements climatiques et résilience des territoires: Quelles leçons en Afrique de l'Ouest, Dakar, King Fahd Palace, 10-12 septembre 2018.

DIEYE E.B. (2007), Les ensembles littoraux de la lagune de Joal-Fadiouth et de l'estuaire du Saloum (Sénégal) : approche méthodologique de la dynamique de mangrove entre 1972 et 2005 par télédétection et système d'information géographique, Thèse de $3^{\text {ème }}$ cycle, Université Cheikh Anta Diop de Dakar, $262 \mathrm{p}$.

DIEYE E.H.B., SANE T., SOLLY B., DIEDHIOU P., BA B.D., THIOR M., MERING C., SY O. \& DIAW A.T. (2021), « Dynamique spatiale et mutations socio-environnementales en zone de mangrove ouestafricaine, de la lagune de Joal-Fadiouth (Sénégal) au Rio de Cacine (Guinée-Bissau) ", Actes du Colloque international LMI-PATEO-UASZ, Université Assane Seck de Ziguinchor (Sénégal), 19-22 novembre 2019, pp. 547-562.

DIOP A. (2017), Évolution de l'occupation des sols et analyse de la disponibilité de la main-d'œuvre rizicole dans le Royaume du Moff-Ewi (Bandial) en Basse Casamance, Mémoire de master de Géographie, Université Assane Seck de Ziguinchor, 94 p.

EHEMBA F.S., LE COQ Y., RUË O., NIOKANE M., BODIVIT M., SECK P., DIALLO M., AGOSSOU S., DESCROIX L., SANE T. \& FABRE M. (2017), « Une cartographie participative pour bâtir une connaissance concertée des mutations de territoire dans l'espace frontalier sénégalo-bissaoguinéen ", in CORMIER-SALEM M.-C., DIAKHATÉ M.M. \& DESCROIX L. (éd.), Sciences participatives et gouvernance des patrimoines et territoires des deltas, Dakar, Karthala, Actes du colloque PATEO/ PRCM, pp. 167-182.

FAYE I.N.B. (2010), Dynamique du trait de côte sur les littoraux sableux de la Mauritanie à la GuinéeBissau (Afrique de l'Ouest) : approches régionale et locale par photo-interprétation, traitement d'images et analyse des cartes anciennes, Thèse de doctorat, Université de Bretagne occidentale, Institut universitaire européen de la mer, 321 p. vol. $1+72$ p. vol. 2 .

GRDR, SANE T., DIEYE E.H.B. \& DESCROIX L. (2017), Un littoral en mouvement : diversité, dynamiques et mutations des territoires frontaliers du sud-ouest du Sénégal et du nord-ouest de la Guinée-Bissau (Oïo, Cacheu, Ziguinchor, Sédhiou), 140 p.

GUICHARD F., KERGOAT L., MOUGIN E., TIMOUK F., BAUP F., HIERNAUX P. \& LAVENU F. (2009), "Surface thermodynamics and radiative budget in the Sahelian Gourma: Seasonal and diurnal cycles », Journal of Hydrology, 375, pp. 161-177.

MENDY V. (2018), Dégradation des agroécosystèmes et problématique de la revitalisation de la riziculture en Basse-Casamance, Thèse de doctorat, Université Assane SECK de Ziguinchor, 248 p. 
NIANG S. (2017), Dégradation chimique et mécanique des terres agricoles du Gandiolais (littoral Nord du Sénégal), analyse des dynamiques actuelles d'adaptation, Thèse de doctorat, Université Gaston Berger de Saint-Louis (Sénégal), 355 p.

PELISSIER P. (1966), Les paysans du Sénégal : les civilisations agraires du Cayor à la Casamance, Paris, Imprimerie Saint-Yrieix, $944 \mathrm{p}$.

PORTERES R. (1962), « Berceaux agricoles primaires sur le continent africain », Journal of African History, 3, 2, pp. 195-210.

SANE T. (2017), Vulnérabilité et adaptabilité des systèmes agraires à la variabilité climatique et aux changements sociaux en Basse-Casamance (Sud-Ouest du Sénégal), Thèse de doctorat de Géographie et Environnement en cotutelle internationale Université Paris Diderot - Paris 7 - Université Cheikh Anta Diop de Dakar, 376 p.

SANE T., CORMIER-SALEM M.-C., DIEYE E.H.B., DESCROIX L., FABRE M., HABERT E., EHEMBA F., BODIVIT M., BA B.D. \& MENDY V. (2017), « La cartographie participative comme outil d'aide à la compréhension des dynamiques territoriales : application sur un terrain de Basse-Casamance (Sénégal) , in CORMIER-SALEM M.-C., DIAKHATÉ M.M., DESCROIX L. (éd.), Sciences participatives et gouvernance des patrimoines et territoires des deltas, Dakar, Karthala, Actes du colloque PATEO/PRCM, pp. 183-196.

SANE T., MERING C., CORMIER-SALEM M.-C., DIEDHIOU I., BA B.D., DIAW A.T. \& TINE A.K. (2018), « Permanences et mutations dans les terroirs rizicoles de Basse-Casamance (Sénégal) », L'Espace Géographique, 47, 3, Belin, pp. 201-218.

SENE C. (2015), Potentialités et limites de la gestion participative des Aires marines protégées : le cas de Joal-Fadiouth, Sénégal, Thèse de doctorat unique, Université de Liège, Faculté des Sciences et de Gestion de l'Environnement, $382 \mathrm{p}$.

SOLLY B., DIEYE E.H.B., SANÉ T. \& DIAW A.T. (2018), « Dynamique de la mangrove de Thiobon dans l'estuaire de la Casamance (Sénégal) entre 1972 et 2017 », European Scientific Journal, 14, 33, pp. 118-133, DOI :10.19044/esj.2018.v14n33p118.

SOUMARE S. (2018), Analyse de la dynamique et de la gestion de la mangrove dans la commune de Kafountine en Basse-Casamance (Sénégal), Mémoire de master, Département de Géographie, Université Assane Seck de Ziguinchor, 165 p.

SULTAN B., LALOU R., SANNI M.A., OUMAROU A. \& SOUMARE M.A. (2015), Les sociétés rurales face aux changements climatiques et environnementaux en Afrique de l'Ouest, Paris, IRD Éditions, Collection Synthèses, $463 \mathrm{p}$.

THIOR M. (2020), Dynamique du littoral de la Casamance : caractéristiques morphodynamique, changements environnementaux et impacts socioéconomique, Thèse de doctorat, Université Assane SECK de Ziguinchor, 389 p.

THIOR M., SANE T., SY O., DESCROIX L., BA B.D., SOLLY B. \& MENDY V. (2019), « Analyse spatiale de l'évolution du trait de côte autour de l'embouchure du fleuve Casamance (Sénégal) de 1968 à 2017, à partir de l'outil DSAS ", European Scientific Journal, 15, 9, pp. 106-130.

\section{NOTES}

1. Instrument aratoire de la plupart des agriculteurs de l'Afrique de l'Ouest à l'image des Diola de Basse-Casamance au Sénégal. 


\section{RÉSUMÉS}

Du fait de sa position géographique (proximité avec l'océan Atlantique), de la richesse de ses écosystèmes (cours d'eau à prédominance de mangrove) et de l'importance des activités socioéconomiques (pêche, riziculture, tourisme, etc.), l'Afrique de l'Ouest demeure une des régions les plus importantes du monde en termes de biodiversité. Elle est parmi les régions les plus touchées par les changements environnementaux et les dynamiques rurales. En s'appuyant, pour l'essentiel, sur les résultats obtenus dans le cadre du consortium Patrimoines et Territoires de l'Eau (PATEO), ce travail analyse la vulnérabilité et la résilience des socio-écosystèmes littoraux d'Afrique de l'Ouest en termes d'état de connaissances actuelles et s'interroge sur leur devenir. Les résultats ont révélé des mutations environnementales et socio-économiques relativement importantes, positives ou négatives, témoignant d'une forte dynamique des zones littorales ouest-africaines. Ces changements reposent sur diverses forces externes et internes qui s'exercent sur les écosystèmes et les sociétés, surtout rurales, et les font évoluer depuis les années 1970. Dans ce contexte, sociétés et milieux sont soumis aux mêmes aléas, mais se sont montrés plus ou moins vulnérables ou résilients en fonction de leur degré de dépendance à l'extérieur. Cette situation influe grandement sur les politiques publiques d'aménagement du territoire.

Due to its geographical position (proximity to the Atlantic Ocean), the richness of its ecosystems (predominantly mangrove rivers) and the importance of socio-economic activities (fishing, rice growing, tourism, etc.), West Africa remains one of the most important regions in the world. It is among the regions most affected by environmental changes and rural dynamics. Basically based on the results obtained within the framework of the Water Heritage and Territories consortium (PATEO), this work analyzes the vulnerability and resilience of coastal socio-ecosystems in West Africa in terms of the state of current knowledge and questions their future. The results revealed relatively significant environmental and socio-economic changes, positive or negative, testifying to the strong dynamics of West African coastal areas. These changes are based on various external and internal forces exerted on ecosystems and societies, especially rural ones, and have been causing them to evolve since the 1970s. In this context, societies and environments are subject to the same hazards, but have shown themselves to be more or less vulnerable or resilient depending on their degree of dependence on the outside. This situation greatly influences public land use planning policies.

\section{INDEX}

Keywords : vulnerability, resilience, socio-ecosystems, coastline, West Africa

Mots-clés : vulnérabilité, résilience, socio-écosystèmes, littoral, Afrique de l'Ouest

\section{AUTEURS}

\section{TIDIANE SANÉ}

Département de Géographie, UFR Sciences et Technologies, Université Assane Seck de Ziguinchor, Sénégal, Laboratoire Mixte International (LMI) « Patrimoines et Territoires de l'Eau (PATEO) », tsane@univ-zig.sn 


\section{EL HADJI BALLA DIÈYE}

Département de Géographie, UFR Sciences et Technologies, Université Assane Seck de Ziguinchor, Sénégal, Laboratoire Mixte International (LMI) « Patrimoines et Territoires de l'Eau (PATEO) », edieye@univ-zig.sn

\section{BOUBACAR SOLLY}

Département de Géographie, UFR Sciences et Technologies, Université Assane Seck de Ziguinchor, Sénégal, Laboratoire Mixte International (LMI) « Patrimoines et Territoires de l'Eau (PATEO) », bakisolly@hotmail.com

\section{BOUBACAR DEMBA BA}

Département de Géographie, UFR Sciences et Technologies, Université Assane Seck de Ziguinchor, Sénégal, Laboratoire Mixte International (LMI) « Patrimoines et Territoires de l'Eau (PATEO) », badembaba@gmail.com

\section{MAMADOU THIOR}

Département de Géographie, UFR Sciences et Technologies, Université Assane Seck de Ziguinchor, Sénégal, Laboratoire Mixte International (LMI) « Patrimoines et Territoires de l'Eau (PATEO)», thioryaz@yahoo.fr

\section{LUC DESCROIX}

Laboratoire Mixte International (LMI) « Patrimoines et Territoires de l'Eau (PATEO) », UMR 208, « Patrimoines Locaux et Gouvernance », IRD, MNHN, Paris, France, luc.descroix@ird.fr

\section{MARIE-CHRISTINE CORMIER-SALEM}

Laboratoire Mixte International (LMI) « Patrimoines et Territoires de l'Eau (PATEO) », UMR 208, «Patrimoines Locaux et Gouvernance », IRD, MNHN, Paris, France, marie.cormier@ird.fr

\section{MOUHAMADOU MAWLID DIAKHATÉ}

Laboratoire Mixte International (LMI) « Patrimoines et Territoires de l'Eau (PATEO) », Laboratoire LEIDI, Université Gaston Berger de Saint-Louis, Sénégal, mdiakhate10@yahoo.com 